\title{
On the contribution of perceptual fluency and priming to recognition memory
}

\author{
M. A. CONROY \\ University of California, San Diego, La Jolla, California \\ R. O. HOPKINS \\ Brigham Young Unversity, Provo, Utah \\ and LDS Hospital, Salt Lake City, Utah \\ and \\ L. R. SQUIRE \\ University of Califormia, San Diego, La Jolla, California \\ and Veterans Affairs Healthcare System, San Diego, California
}

\begin{abstract}
Repetition priming has been shown to be independent of recognition memory. Thus, the severely amnesic patient E.P. has demonstrated intact stem completion priming and perceptual identification priming, despite at-chance performance on recognition memory tasks. It has also been shown that perceptual fluency can influence feelings of familiarity, in the sense that items perceived more quickly tend to be identified as familiar. If studied items are identified more fluently, due to perceptual priming, and fluency leads to familiarity, why do severely amnesic patients perform no better than chance on recognition memory tasks? One possibility is that severely amnesic patients do not exhibit normal fluency. Another possibility is that fluency is not a sufficiently strong cue for familiarity. In two experiments, 2 severely amnesic patients, 3 moderately amnesic patients, and 8 controls saw words slowly clearing from a mask. The participants identified each word as quickly as possible and then made a recognition (old/new) judgment. All the participants exhibited fluency, in that old responses were associated with shorter identification times than new responses were. In addition, for the severely amnesic patients, priming was intact, and recognition memory performance was at chance. We next calculated how much priming and fluency should elevate the probability of accurate recognition. The tendency to identify studied words rapidly (.6) and the tendency to label these rapidly identified words old (.6) would result in $36 \%$ of the studied words being labeled old. Other studied words were identified slowly (.4) but were still labeled old (.4), resulting in an additional $16 \%$ of studied words labeled old. Thus, the presence of fluency increases the probability of accurate recognition judgments to only $52 \%$ (chance $=50 \%$ ). This finding explains why amnesic patients can exhibit both priming and fluency yet still perform at chance on recognition tests.
\end{abstract}

Declarative memory supports the capacity for conscious recollection of facts and events (Eichenbaum \& Cohen, 2001; Squire, 1992). One of the most widely studied examples of declarative memory is recognition, the ability to judge items as having been previously encountered. Declarative memory can be contrasted with a collection of nondeclarative memory abilities, including skill learning, simple forms of conditioning, and the phenomenon of priming (Knowlton \& Packard, 2002; Schacter \& Tulving, 1994; Squire, 2004). Priming refers to an improved ability to produce or identify an item on the basis of a recent en-

This work was supported by the Medical Research Service of Veterans Affairs, National Institute of Mental Health Grant MH24600, and the Metropolitan Life Foundation. We thank Jennifer Frascino for assistance in data collection and Craig Stark and John Wixted for advice. Correspondence concerning this article should be addressed to L. R. Squire, VAMC (116A), 3350 La Jolla Village Drive, San Diego, CA 92161 (e-mail: 1squire@ucsd.edu). counter with the same or a related item (Schacter \& Buckner, 1998; Tulving \& Schacter, 1990).

There has been extended exploration of the possibility that priming and recognition memory might be related in some way - for example, that priming might lead to a feeling of familiarity and, thereby, might influence recognition judgments. For example, it has been proposed that previously encountered stimuli are processed more fluently and that the fluency with which items are processed is an important basis for judgments of familiarity (Mandler, 1980). Similarly, it has been suggested that a previously studied item is likely to "jump out from the page," in the sense that it is more readily perceived than other items, with the result that the item seems familiar (Jacoby \& Dallas, 1981). The idea is that the familiarity of an item is based, in part, on an unconscious process whereby the fluency of processing is attributed to a previous encounter with the item.

Direct evidence for such an effect comes from studies in which the fluency of processing has been varied. Indi- 
viduals who were asked to identify words partially occluded by a mask were more likely to judge that a word had been encountered recently when identification was easy than when identification was difficult (Johnston, Hawley, \& Elliott, 1991; Whittlesea, 1993; Whittlesea, Jacoby, \& Girard, 1990). Similarly, both moderately severe amnesic patients and controls relied on fluency to make recognition judgments when declarative memory for words was unavailable (Verfaellie \& Cermak, 1999). These findings indicate that fluency can affect recognition memory judgments.

If previously encountered words are perceived more easily due to intact priming and if easily perceived items seem familiar due to fluency, priming should cause previously encountered words to seem more familiar. Accordingly, whenever individuals exhibit priming for previously encountered items, they should also recognize these items as familiar. Because amnesic patients exhibit impaired recognition memory but, nevertheless, can perform above chance, it has been an interesting possibility that they use the fluency of primed items as a cue for familiarity and that intact fluency accounts for why recognition performance is above chance (Verfaellie \& Cermak, 1999; Verfaellie \& Keane, 2002).

A difficulty with this view is that patients with large medial temporal lobe lesions and profound memory impairment can perform at chance on conventional recognition memory tests, despite exhibiting intact perceptual and conceptual priming (Hamann \& Squire, 1997; Levy, Stark, \& Squire, 2004; Stark \& Squire, 2000). There appear to be two ways to understand these findings. One possibility is that severely amnesic patients do not exhibit fluency and that, therefore, they do not have a tendency to judge easily perceived items as familiar. Another possibility is that the impact of priming and fluency is too weak to measurably elevate recognition accuracy. To explore these possibilities, we asked whether severely amnesic patients exhibit normal fluency and, if so, how strong an influence should priming and fluency be expected to have on recognition memory performance.

In Experiment 1, a method was adopted that was used previously to demonstrate perceptual fluency in patients with moderately severe memory impairment (Verfaellie $\&$ Cermak, 1999). The influence of declarative memory was eliminated by the use of a mock-subliminal study phase in which no words were actually presented. Next, during the test phase, the participants identified words as they gradually cleared from a mask and then judged whether the words had been presented previously. The question of interest was whether severely amnesic patients would exhibit fluency - that is, would their recognition judgments be influenced by how fluently they processed items at test.

\section{EXPERIMENT 1}

\section{Method}

Participants. We tested two male patients (E.P. and G.P.) who have large medial temporal lobe lesions and severe amnesia as the result of herpes simplex encephalitis (MTL group). Three additional male patients (G.W., J.R.W., and R.S.) with damage limited to the hippocampal region and less severe amnesia were also tested (H group).

E.P. and G.P. have been described in detail elsewhere (Bayley \& Squire, 2005). Briefly, E.P. was born in 1922, has 12 years of education, and developed amnesia in 1992. His lesion includes the amygdala, the hippocampal region, the entorhinal cortex, the perirhinal cortex, much of the parahippocampal cortex, the anterior fusiform gyrus, and the anterior insula. G.P. was born in 1946, has 16 years of education, and developed amnesia in 1987. Like E.P., G.P.'s lesion is primarily medial temporal, but his lesion extends more laterally. The damage includes, bilaterally, the amygdala, the hippocampal region, the entorhinal cortex, the perirhinal cortex, much of the parahippocampal cortex, the anterior fusiform gyrus, the anterior insula, and the anterior $1 \mathrm{~cm}$ of the lateral temporal lobe.

G.W. was born in 1959, has 12 years of education, and became amnesic in 2001 after respiratory failure associated with a drug overdose. J.R.W. was born in 1963, has 12 years of education, and became amnesic in 1990 after an ischemic episode associated with cardiac arrest. R.S. was born in 1956, has 12 years of education, and became amnesic in 1998 after respiratory failure associated with a drug overdose. Relative to controls (19 males; mean age, 49 years), G.W., J.R.W., and R.S. have an average bilateral reduction in hippocampal volume of $48 \%, 44 \%$, and $33 \%$, respectively. The parahippocampal gyrus was of normal size (mean reduction, $6 \%$; Gold \& Squire, 2005). For behavioral testing, 8 controls were matched to the MTL group (mean age, 74 years; mean education, 12.4 years).

Materials and Procedure. Eighty six-letter words with a frequency of 8-21 per million (Kučera \& Francis, 1967) served as stimuli. Ten similar words were used during practice trials at the beginning of the test phase. In the study phase, a visual mask $(3.0 \times$ $0.8 \mathrm{~cm}$ rectangle; Figure 1A) was presented on a computer screen on each of 40 mock study trials (exposure time of mask $=80 \mathrm{msec}$,
A Experiment 1 Study:

Test:
7.M. DIVIDE;

Time (sec): 0
B Experiment 2 Study: PRAISE Test:
W PRAISE

3.5

7.0

10.5

Figure 1. Sample stimuli. (A) In Experiment 1, only a mask was presented on each trial during the study phase (no words). At test, words gradually cleared from the mask over 11 sec. (B) In Experiment 2, words were presented for 2 sec during the study phase. At test, words were presented as in Experiment 1. 
intertrial interval $=1 \mathrm{sec}$ ). The participants were told that 40 words were presented, but too quickly for conscious perception (no words were actually presented).

In the test phase, the participants identified words as quickly as possible as they gradually cleared from a mask (Figure 1A) and then decided whether the words had appeared at study. Each of 80 trials began with a word obscured by the mask (440 small black squares covering $70 \%$ of a $3.0 \times 8.0 \mathrm{~cm}$ rectangle). Blocks were gradually removed (4 squares per $100 \mathrm{msec}$ ) until the participants halted the trial with a barpress and identified the word. If the word was identified correctly, the remainder of the mask was cleared, and the participants decided (yes/no) whether the word had appeared during the mock study phase. If the word was misidentified (this occurred on only 3.0 trials/participant), the mask continued to clear until the participant identified the word. The participants were told that half of the words had appeared during the study phase.

\section{Results}

When the participants were asked to decide whether the words they identified had appeared during the study phase (Figure 2), they exhibited the fluency effect. Thus, words that were labeled old were identified more quickly than words that were labeled new (MTL patients, words labeled old were identified in $9,119 \mathrm{msec}$, and words labeled new were identified in $9,998 \mathrm{msec}$; H patients, words labeled old were identified in 7,464 msec, and words labeled new were identified in $7,907 \mathrm{msec}$; CON group, words labeled old were identified in $8,719 \mathrm{msec}$, and words labeled new were identified in $9,408 \mathrm{msec}$ ). An analysis of variance (ANOVA; labeled old vs. labeled new $\times$ group) yielded a marginal effect of group $(p=$ .061 ), due to the faster overall response times for the $\mathrm{H}$ patients, who were younger than the participants in the other two groups. Importantly, there was an effect of response type $[F(1,10)=10.97, p<.01]$ but no interaction of response type and group $(p>.7)$. Thus, the identifi-

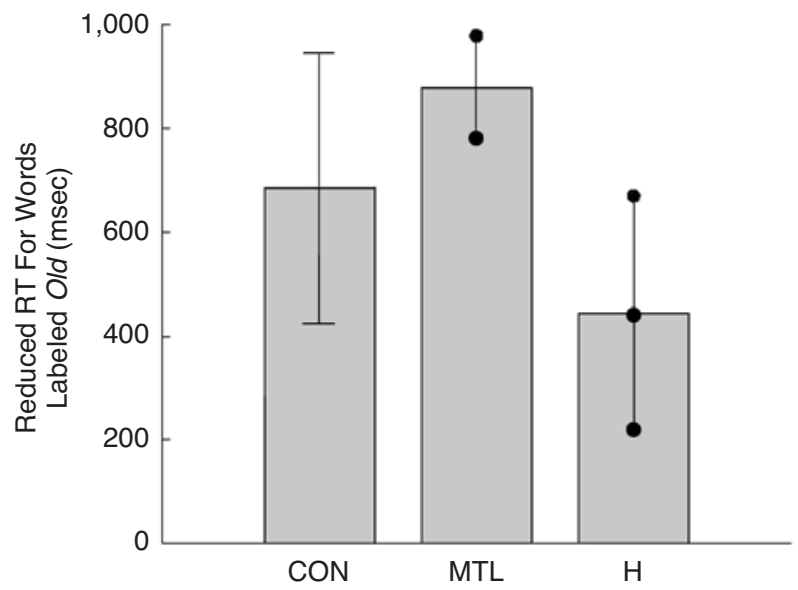

Figure 2. Magnitude of the fluency effect (identification time for words labeled new minus identification time for words labeled old) in Experiment 1 for controls ( $\mathrm{CON}, n=8)$, patients with large medial temporal lobe lesions (MTL, $n=2$ ), and patients with damage limited to the hippocampal region $(\mathrm{H}, n=3)$. Circles show the individual scores for the patients. Bracket shows standard errors of the means. cation times for words labeled old were shorter than the identification times for words labeled new.

The magnitude of the fluency effect can also be measured as the increased likelihood of labeling words old when they were identified quickly. When each word was categorized as quickly or slowly identified (relative to a participant's median response time), the words identified quickly were more likely to be labeled old than were the words identified slowly. Thus, MTL patients labeled as old $61.3 \%$ of the words that they identified quickly but only $35.0 \%$ of the words that they identified slowly. For $\mathrm{H}$ patients, these values were $59.2 \%$ and $50.0 \%$; for the CON group, these values were $64.4 \%$ and $48.4 \%$. An ANOVA (fast or slow response time $\times$ group) yielded an effect of response time $[F(1,10)=17.47, p<.01]$ but no effect of group $(p>.6)$ and no interaction of response time and group $(p>.4)$.

\section{EXPERIMENT 2}

The fact that patients known to have severely impaired recognition memory (the MTL group) nevertheless exhibited as great a fluency effect as the controls challenges the idea that fluency leads to improved recognition. However, it is possible that the mock study task used in Experiment 1 allows fluency to support subsequent recognition judgments more effectively than would traditional recognition memory tasks. To test this possibility, we followed the method of Verfaellie and Cermak (1999) and repeated Experiment 1, but now with presentation of half the test words during the study phase.

\section{Method}

Participants. The participants were the same as those in Experiment 1.

Materials and Procedure. As in Experiment 1, 80 six-letter words with a frequency of 8-21 per million (Kučera \& Francis, 1967) served as stimuli. Forty of these words were presented during the study phase (Figure 1B; exposure time $=2 \mathrm{sec}$; intertrial interval $=3 \mathrm{sec}$ ). Ten similar words were used during practice trials at the beginning of the test phase.

The test phase was identical to that in Experiment 1, in that 80 words were presented ( 40 studied words and 40 new words), and the participants identified the words as quickly as possible as they gradually cleared from the mask (Figure 1B). If the word was identified correctly, the remainder of the mask was cleared, and the participant decided (yes/no) whether the word had appeared during the study phase. If the word was misidentified (this occurred on only 1.1 trials/participant), the mask continued to clear until the participant identified the word.

\section{Results}

When the participants were asked to identify words as they cleared from a mask (Figure 3A), they exhibited priming. Thus, the studied words were identified more quickly than the unstudied words (MTL patients, 8,929 msec for the studied words vs. $9,436 \mathrm{msec}$ for the unstudied words; $\mathrm{H}$ patients, 7,058 msec vs. 7,637 msec; CON group, $8,260 \mathrm{msec}$ vs. $8,813 \mathrm{msec}$ ). An ANOVA (studied vs. unstudied words $\times$ group) yielded an effect of word type $[F(1,10)=92.18, p<.001]$, a marginal effect of group 

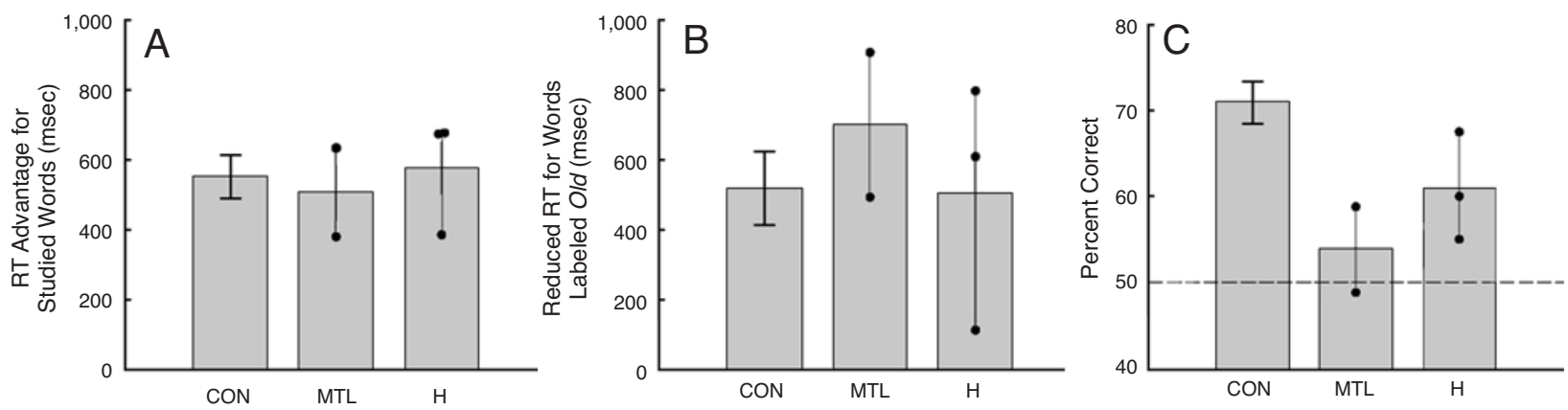

Figure 3. (A) Magnitude of the priming effect (identification time for unstudied words minus identification time for studied words) in Experiment 2 for controls (CON, $n=8$ ), patients with large medial temporal lobe lesions (MTL, $n=2)$, and patients with damage limited to the hippocampal region $(\mathrm{H}, n=3)$. (B) Magnitude of the fluency effect in Experiment 2 (identification time for words labeled new minus identification time for words labeled old). (C) Percentage of correct responses (hits plus correct rejections) on a test of recognition memory. Circles show individual scores for the patients. Dashed line indicates chance performance. Bracket shows standard errors of the means.

$(p=.10)$ due to faster response times for the $\mathrm{H}$ group, and no interaction of word type and group $(p>.9)$.

The participants also exhibited the fluency effect (Figure 3B). Thus, words that were judged as having appeared during the study phase were identified more quickly than words that were judged to be new (MTL patients, words labeled old were identified in $8,881 \mathrm{msec}$, and words labeled new were identified in $9,581 \mathrm{msec} ; \mathrm{H}$ patients, words labeled old were identified in $7,140 \mathrm{msec}$ vs. words labeled new in 7,646 msec; CON group, words labeled old were identified in $8,266 \mathrm{msec}$ vs. words labeled new in 8,786 msec). An ANOVA (labeled old vs. labeled new $\times$ group) yielded a marginal effect of group ( $p=.08$; as in Experiment 1 , the younger $\mathrm{H}$ group responded more quickly overall), an effect of response type $[F(1,10)=32.98, p<.001]$, and no interaction of response type and group $(p>.7)$.

When the data for the studied and the unstudied words were analyzed separately, the effect of response type was evident for the studied words $[F(1,10)=33.65, p<$ $.001)$, but not for the unstudied words $[F(1,10)=1.71$, $p>.2]$. Most likely, at least for the controls in Experiment 2, response speed, as well as old/new decisions, were influenced primarily by the availability of declarative memory for previously encountered words. Indeed, the 8 controls demonstrated no tendency for unstudied words that were labeled old to be identified more quickly than unstudied words labeled new $(8,839 \mathrm{msec}$ vs. $8,831 \mathrm{msec})$. In contrast, the 2 severely amnesic patients did exhibit this effect $(9,180 \mathrm{msec}$ vs. 9,706 msec), just as they did in Experiment 1 .

The fluency effect was also evident as an increased likelihood to label old the words that were identified quickly - that is, more quickly than a participant's median response time. Thus, the MTL patients labeled old $66.3 \%$ of the words that they identified quickly but only $46.2 \%$ of the words that they identified slowly. For the $\mathrm{H}$ patients, these values were $62.5 \%$ and $49.0 \%$; for the CON group, these values were $57.8 \%$ and $38.7 \%$. An
ANOVA (short or long response time $\times$ group) yielded an effect of response time $[F(1,10)=28.91, p<.001]$ but no effect of group $(p>.4)$ and no interaction of response time and group $(p>.6)$.

Figure 3C shows each group's recognition memory performance (MTL patients, 53.8\% correct, $d^{\prime}=0.19 ; \mathrm{H}$ patients, $60.8 \%$ correct, $d^{\prime}=0.59$; CON group, $71.3 \%$ correct, $\left.d^{\prime}=1.31\right)$. Both the H group and the MTL group performed more poorly than the CON group $(p s<.03)$. The MTL group did not perform above chance $(p=.6)$, and the $\mathrm{H}$ group performed marginally above chance $(p=.1)$.

\section{GENERAL DISCUSSION}

In two experiments, memory-impaired patients, including 2 severely impaired patients with large medial temporal lobe lesions, identified 80 words as they cleared from a mask and then made yes-no recognition memory judgments for the words that they had identified. In Experiment 1 , these tests were preceded by a mock study phase in which no words were actually presented. In Experiment 2 , the tests were preceded by a study phase involving 40 words that also appeared in the test phase. In both experiments, words that were identified quickly tended to be labeled as having been presented previously, and words that were identified slowly tended to be labeled $n e w$. The magnitude of these effects was quite similar across groups, although of course the small number of participants limits the power of between-group comparisons. These findings demonstrate that even severely amnesic patients exhibit the effects of perceptual fluency on recognition memory judgments. In addition, in Experiment 2 , the identification time for the studied words was shorter than the identification time for the unstudied words. That is, the participants exhibited perceptual priming. Finally, the amnesic patients were impaired on the recognition memory test, and the patients with large medial temporal lobe lesions performed at chance. 
These results appear, on first consideration, to contain a contradiction. If studied words are identified more fluently as a result of priming and fluency leads to familiarity, how is it that severely amnesic patients performed no better than chance on the recognition task? The present findings rule out one possible explanation of this outcome-namely, that severely amnesic patients do not exhibit fluency effects. Indeed, the MTL patients exhibited fluency effects in both Experiments 1 and 2 that on average were, if anything, greater than the fluency effect exhibited by controls. A second possible explanation is that priming and fluency exert only a weak effect on recognition memory judgments. To explore how strong an influence priming and fluency should be expected to have on recognition performance, we first asked how large an effect would occur if the participants were able to use the cues provided by priming in a fully efficient way (i.e., if the participants labeled all the words identified more quickly than the median response time old and all the words identified more slowly than the median response time $n e w)$. This calculation provides an estimate of the maximum potential contribution of priming and fluency to familiarity judgments.

This question was explored in an earlier study that calculated the potential benefit of priming in the context of a speeded lexical decision task (Poldrack \& Logan,
1997). In that study, participants made a recognition judgment immediately after each lexical decision and, potentially, could have used the speed of their lexical decision as a basis for judging familiarity (lexical decisions were faster for previously studied words). In this case, if response speed had been used as a strict guide for recognition judgments, recognition accuracy would have been only slightly above chance ( $d^{\prime}$ would have ranged from 0.14 to 0.38 across experimental conditions). In contrast, actual recognition performance was much better $\left(d^{\prime}\right.$ ranged from 1.29 to 2.46 ). Thus, in that study processing fluency (as indexed by response speed) could not have been a large factor in recognition judgments.

Like Poldrack and Logan (1997), we also calculated to what extent response speed could have influenced recognition accuracy in our study if response speed (identification time) was used as a perfect cue. In Experiment 2 , the median response time averaged $8,441 \mathrm{msec}$ for the CON group, 7,249 msec for the H group, and $9,050 \mathrm{msec}$ for the MTL group. If all the words that were identified more quickly than a participant's median response time were labeled old and if all the words that were identified more slowly than the median response time were labeled new, the participants would have achieved an average recognition score of $60.2 \%$ correct. Specifically, accuracy would have been $61.6 \%\left(d^{\prime}=0.59\right)$ for the CON

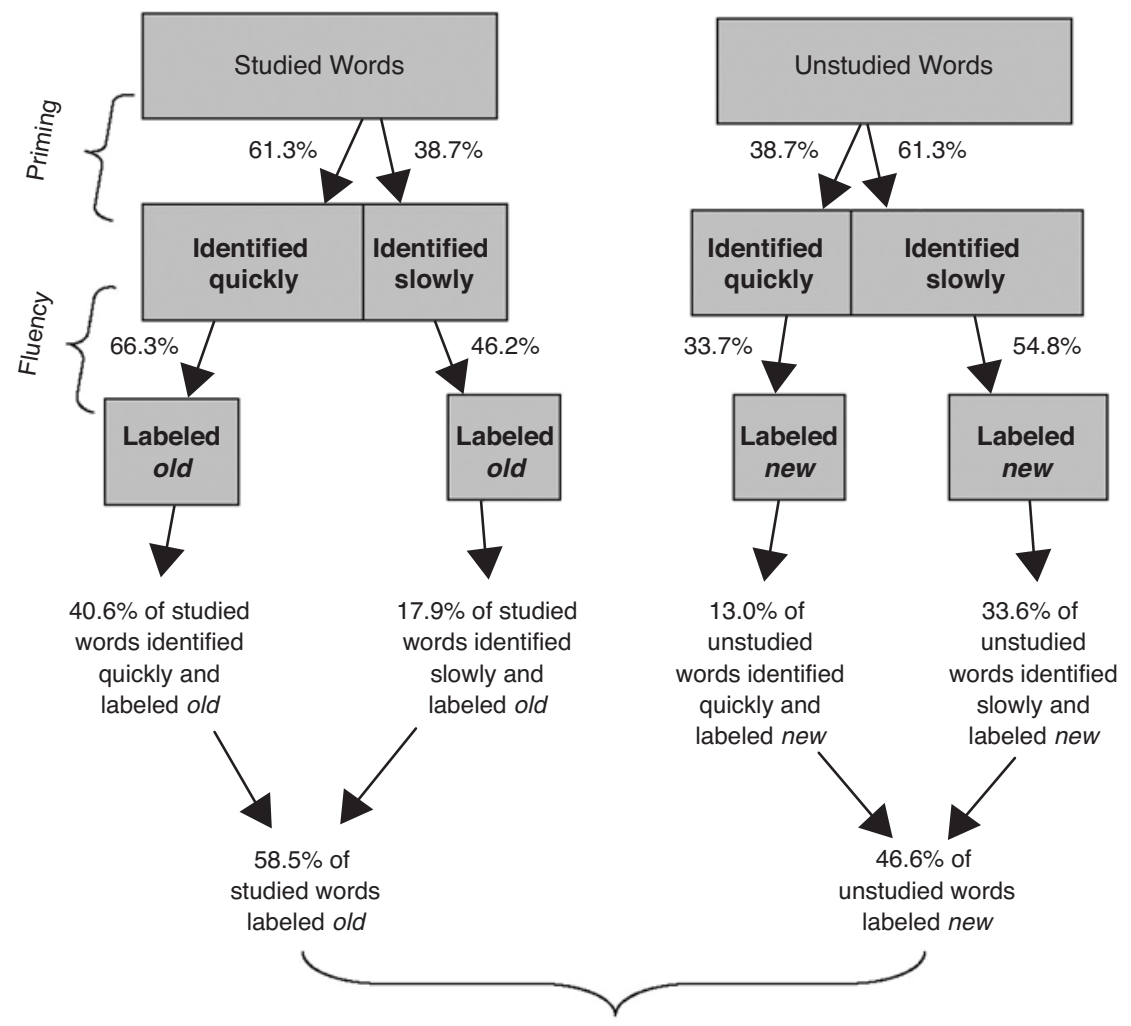

Expected recognition performance $=\mathbf{5 2 . 6} \%$

Figure 4. Derivation of the recognition score that would be expected for the MTL group, given the observed magnitude of priming and fluency (see the text). 
group, $55.8 \%\left(d^{\prime}=0.29\right)$ for the H group, and $61.3 \%$ $\left(d^{\prime}=0.57\right)$ for the MTL group. Thus, even under ideal conditions, identification time could not have been a strong cue for recognition memory accuracy.

The more interesting question is to ask how large an effect on recognition memory would actually be expected, given the observed magnitude of the fluency effect within each group for studied and unstudied words. Figure 4 illustrates the calculations used to answer this question. Thus, the MTL patients identified $61.3 \%$ of the studied words quickly, and of all the words that they identified quickly, $66.3 \%$ were labeled old. These two effects predict that $40.6 \%$ of studied words should be both identified quickly and labeled old $(.613 \times .663)$. The remainder of the studied words were identified slowly $(38.7 \%)$, and a minority of the slowly identified words $(46.2 \%)$ were, nevertheless, labeled old, resulting in $17.9 \%$ of the studied words that should both be identified slowly and also correctly labeled old $(.387 \times$ .462). These calculations indicate that the MTL patients should label $58.5 \%$ of the studied words accurately $(40.6 \%+17.9 \%)$.

A similar calculation for unstudied words begins with the observation that most of the unstudied words were identified slowly (61.3\%) and that $54.8 \%$ of the slowly identified words were labeled new. Thus, $33.6 \%$ of the unstudied words should both be identified slowly and labeled new $(.613 \times .548)$. The remaining unstudied words $(38.7 \%)$ were identified quickly, and $33.7 \%$ of these were nevertheless labeled new, with the result that $13.0 \%$ of the unstudied words should be identified quickly and also correctly labeled new. These calculations indicate that the MTL patients should label $46.6 \%$ of the unstudied words accurately $(33.6 \%+13.0 \%)$. Averaging the accuracy for the studied $(58.5 \%)$ and the unstudied $(46.6 \%)$ words predicts that recognition accuracy for the MTL group should be $52.6 \%$. Similar calculations yield predictions of $50.8 \%$ accuracy for the $\mathrm{H}$ group and $52.2 \%$ accuracy for the CON group. Thus, priming and fluency do contribute to recognition accuracy, but only slightly, and not to a level that is discernibly different from chance.

Another way of understanding this conclusion is to note that natural variations in fluency overshadow the variation in fluency that arises from having previously encountered some of the test items. The mean difference in response times at test for the studied and the unstudied words was $551 \mathrm{msec}$ across all participants. Yet the range of response times for an individual participant typically spanned $4-5 \mathrm{sec}$. Accordingly, there were many influences on response time besides the influence that came from a recent encounter with a test item. As a result, under the conditions of our studies, fluency was not a reliable source of information about recent encounters. It remains possible, of course, that conditions might be found in which variation in fluency is primarily a function of prior encounters, rather than of other factors, and in this circumstance fluency might measurably influence recognition performance. At the same time, one would expect that it would be difficult to achieve such conditions in more typical learning situations, where items are presented "all at once," rather than gradually (as in our studies). Priming effects are typically larger when test words appear gradually (Johnston et al., 1991; Ostergaard, 1999).

Fluency not only can bias recognition judgments, albeit without appreciably improving accuracy, but also can influence perceptual judgments about the characteristics of an item (Whittlesea, 1993; Whittlesea et al., 1990). Thus, fluency is thought to result in an unconscious attribution process that can influence the perception of an item's meaning, pleasantness, or duration (Whittlesea, 1993). For example, words that are pronounced more quickly are rated as more pleasant and more related in meaning to a recently presented word. Fluency can best be viewed as providing a perceptual bias, rather than accurate information about previous encounters. This bias is indifferent to whether an item was studied or not and is based on whether an item is identified quickly or slowly. Interestingly, healthy (nonamnesic) individuals appear to rely on fluency only when declarative memory is weak (Johnston et al., 1991; Verfaellie \& Cermak, 1999).

In light of the negligible contribution of perceptual fluency to recognition accuracy, it is useful to consider the possibility that conceptual fluency is an important cue for familiarity (Rajaram \& Geraci, 2000; Verfaellie \& Keane, 2002; Whittlesea, 1993). Conceptual fluency refers to the ease with which the meaning of an item is processed. If recognition memory accuracy can be supported significantly by conceptual fluency, patients exhibiting intact conceptual priming should also perform above chance on recognition memory tests. Yet E.P. and G.P. exhibited fully intact conceptual priming during free-association tests and category verification tests but performed at chance on recognition memory tests involving the same words as those used to demonstrate priming (Levy et al., 2004). These findings suggest that conceptual fluency is not a useful source of information about familiarity.

The conclusion that fluency is not a reliable cue for familiarity judgments is consistent with other findings. Patients with occipital lobe lesions can exhibit impaired perceptual priming but perform normally at tasks of recognition memory (Gabrieli, Fleischman, Keane, Reminger, \& Morrell, 1995; Keane, Gabrieli, Mapstone, Johnson, \& Corkin, 1995). In addition, in fMRI, recognition and priming were associated with distinct patterns of increased and decreased activity, respectively (Donaldson, Petersen, \& Buckner, 2001). Finally, recognition memory and perceptual priming were sharply dissociable in behavioral studies of healthy volunteers (Wagner, Gabrieli, \& Verfaellie, 1997).

In summary, in the present study, we examined the phenomenon of perceptual fluency and priming in amnesic 
patients and the relationship of fluency and priming to recognition memory. Although the patients exhibited intact fluency - that is, they exhibited the normal influence of response speed on familiarity judgments - and although they exhibited intact priming, the severely amnesic patients exhibited chance recognition memory performance. Further consideration of how large an effect on recognition memory should be expected, given the strength of the fluency effect, revealed that the effect of fluency on accuracy was weak and that fluency would not be expected to elevate recognition scores to a level discernibly different from chance. These findings explain why severely amnesic patients can exhibit both priming and fluency yet still perform at chance on recognition tests. In addition, less severely impaired patients who are able to perform above chance on recognition memory tests are more likely relying on their residual declarative memory, rather than on fluency, as a cue for familiarity.

\section{REFERENCES}

BAyley, P., \& SQuire, L. R. (2005). Failure to acquire new semantic knowledge in patients with large medial temporal lobe lesions. Hippocampus, 15, 273-280.

Donaldson, D. I., Petersen, S. E., \& Buckner, R. L. (2001). Dissociating memory retrieval processes using fMRI: Evidence that priming does not support recognition memory. Neuron, 31, 1047-1059.

Eichenbaum, H., \& Cohen, N. J. (2001). From conditioning to conscious recollection: Memory systems of the brain. New York: Oxford University Press.

Gabrieli, J. D. E., Fleischman, D. A., Keane, M. M., Reminger, S. L., \& Morrell, F. (1995). Double dissociation between memory systems underlying explicit and implicit memory in the human brain. Psychological Science, 6, 76-82.

GoLD, J. J., \& SQUIRE, L. R. (2005). Quantifying medial temporal lobe damage in memory-impaired patients. Hippocampus, $\mathbf{1 5}, 79-85$.

HamanN, S. B., \& SQuiRe, L. R. (1997). Intact perceptual memory in the absence of conscious memory. Behavioral Neuroscience, $\mathbf{1 1 1}$, 850-854.

JACOBY, L. L., \& DaLlas, M. (1981). On the relationship between autobiographical memory and perceptual learning. Journal of Experimental Psychology: General, 3, 306-340.

Johnston, W. A., Hawley, K. J., \& Elliott, M. G. (1991). Contribution of perceptual fluency to recognition judgments. Journal of Experimental Psychology: Learning, Memory, \& Cognition, 17, 210-223.

Keane, M. M., Gabrieli, J. D. E., Mapstone, H. C., Johnson, K. A., \& CORKIN, S. (1995). Double-dissociation of memory capacities after bilateral occipital-lobe or medial temporal-lobe lesions. Brain, $\mathbf{1 1 8}$, 1129-1148.

KNOWLTON, B. J., \& Packard, M. G. (2002). Learning and memory functions of the basal ganglia. Annual Review of Neuroscience, 25, 563-593.

KUČERA, H., \& FRANCIS, W. N. (1967). Computational analysis of presentday American English. Providence, RI: Brown University Press.

LeVy, D. A., Stark, C. E. L., \& SQuire, L. R. (2004). Intact conceptual priming in the absence of declarative memory. Psychological Science, 15, 680-686.

MANDLER, G. (1980). Recognizing: The judgment of previous occurrence. Psychological Review, 87, 252-271.

OstergaARD, A. L. (1999). Priming deficits in amnesia: Now you see them, now you don't. Journal of the International Neuropsychological Society, 5, 175-190.

PoldRACK, R. A., \& LOGAN, G. D. (1997). Fluency and response speed in recognition judgments. Memory \& Cognition, 25, 1-10.

Rajaram, S., \& Geraci, L. (2000). Conceptual fluency selectively influences knowing. Journal of Experimental Psychology: Learning, Memory, \& Cognition, 26, 1070-1074.

SchaCTER, D. L., \& BuCKNER, R. L. (1998). Priming and the brain. Neuron, 20, 185-195.

SCHACTER, D. L., \& Tulving, E. (EDs.) (1994). Memory systems 1994. Cambridge, MA: MIT Press.

SQuire, L. R. (1992). Memory and the hippocampus: A synthesis from findings with rats, monkeys, and humans. Psychological Review, 99 195-231.

SQUIRE, L. R. (2004). Memory systems of the brain: A brief history and current perspective. Neurobiology of Learning \& Memory, 82, 171177.

STARK, C. E. L., \& SQuire, L. R. (2000). Recognition memory and familiarity judgments in severe amnesia: No evidence for a contribution of repetition priming. Behavioral Neuroscience, 114, 459-467.

Tulving, E., \& Schacter, D. L. (1990). Priming and human memory systems. Science, 247, 301-306.

Verfaellie, M., \& CerMaK, L. (1999). Perceptual fluency as a cue for recognition judgments in amnesia. Neuropsychology, 13, 198-205.

Verfaellie, M., \& Keane, M. M. (2002). Impaired and preserved memory processes in amnesia. In L. R. Squire \& D. L. Schacter (Eds.), Neuropsychology of memory (pp. 35-46). New York: Guilford.

Wagner, A. D., Gabrieli, J. D. E., \& Verfaellie, M. (1997). Dissociations between familiarity in explicit recognition and implicit perceptual memory. Journal of Experimental Psychology: Learning, Memory, \& Cognition, 23, 305-323.

WhitTlesea, B. W. A. (1993). Illusions of familiarity. Journal of Experimental Psychology: Learning, Memory, \& Cognition, 19, 12351253.

Whittlesea, B. W. A., Jacoby, L. L., \& Girard, K. (1990). Illusions of immediate memory: Evidence of an attributional basis for feelings of familiarity and perceptual quality. Journal of Memory \& Language, 29, 716-732. 